VLSI Design

1995, Vol. 3, No. 2, pp. 99-100

Reprints available directly from the publisher

Photocopying permitted by license only (c) 1995 OPA (Overseas Publishers Association)

Amsterdam B.V. Published under license by

Gordon and Breach Science Publishers SA

\title{
Classical and Quantum Hydrodynamic Device Models and Energy Transport
}

\author{
JOSEPH W. JEROME
}

$\mathbf{T}$ The papers in this issue are divided into three natural categories, classical hydrodynamic models, quantum hydrodynamic models, and energy transport models. The classical hydrodynamic models follow a fundamental paper of Bløtekjaer in 1970 in which systems of equations, based upon moments of the Boltzmann equation, were introduced. These equations can be characterized via conservation of particle number, momentum, and energy. The quantum hydrodynamic equations, proceeding from a Wigner function formulation, and containing quantum corrections, were introduced by Iafrate, Grubin, and Ferry in 1981. The energy transport models originate from Stratton's approximation (1962). Of the eight papers included in this issue, four are in the first grouping, three in the second, and one in the final category.

An important paper by Schur in 1976 demonstrated how velocity overshoot could be modeled by momentum and energy balance equations, with appropriate relaxation expressions; this gave impetus to self-consistent models extending drift-diffusion, and likely accounts for the intense study of hydrodynamic models in the subsequent years. The principal features of these models reside in the momentum relaxation time parametrization and the closure of the third moment by a constitutive relation for the heat flux. In an important paper published in 1990, Gnudi, Odeh, and Rudan documented the sensitivity of the heat conduction term in the hydrodynamic model. They analyzed the spurious overshoot detected when the Fourier law, coupled with the Wiedemann-Franz law for the conductivity coefficient, is used to model the heat flux. A somewhat "ad hoc" selection of the constant was required to align the simulation results with those obtained by Monte-Carlo methods. It is now known that this choice is also structure dependent.

The papers in this section of the issue deal with various aspects of these questions. The papers by Ancona and by Rudan and Baccarani give alterna- tive approaches, loosely termed macroscopic and microscopic, to a consistent derivation of the hydrodynamic system. Ancona's approach begins with classical field theory, while Rudan and Baccarani study the subtle aspects of moment closure. These papers are intended to provide a proper theoretical foundation for the classical models. Ancona suggests that the important case when the heat conduction term is not significant leads to a situation where the energy equation need not be solved independently. On the other hand, terms of this type are fundamental to the analysis of Rudan and Baccarani. The final two papers in this section describe two of the modern shock capturing algorithms, which were originally developed for gas dynamics. The paper by Jerome and Shu makes use of the ENO (essentially nonoscillatory) algorithm to determine the effect of a more elaborate heat flux, including a convective term, originally suggested by Stettler, Alam, and Lundstrom (1993). This algorithm was developed at UCLA in the 1980s. The paper also studies the surprising effect of doping dependent mobility functions on device characteristics. The paper by Chen, Cockburn, Jerome, and Shu implements a discontinuous Galerkin finite element method to simulate two dimensional MESFET devices. This algorithm was analyzed by Cockburn and Shu. Both algorithms are capable of dealing with the important case when inertial effects cannot be neglected. In particular, the hydrodynamic model has hyperbolic modes, and numerical procedures must be capable of detecting associated shocks or steep fronts.

The quantum model includes perturbations of the standard pressure tensor and energy density, and is able to simulate effects in small semiconductor devices, including tunnel diodes. The paper of Zhou and Ferry is a review of one such approach. It includes the derivation of the perturbations via a pure state approach. Barrier penetration and repulsion are studied for HEMT devices; the effect of the quantum correction on density smoothing is clearly 
explained. This has an interesting mathematical interpretation as the occurrence of distributions in the conservation equations, arising from the differentiation of discontinuous potentials.

A perturbation involving a contraction factor of $\frac{1}{3}$ in the quantum potential is used by Grubin and Kreskovsky, who also analyze the effect of the contraction. Two dimensional HEMT structures are also studied. These authors employ the Wigner perturbation in the energy density, also derived by Ancona and Iafrate (1989), based on a potential expansion. This perturbation was also used by Gardner in his paper, which describes multiple regions of negative differential resistance in a resonant tunneling diode, and provides an accompanying wave function phase analysis.

Models of energy transport type complete our presentation. Such models have been introduced to take advantage of microscopic relaxation and nonparabolic energy bands. The paper of Kan, Chen, Ravaioli, Yu, and Dutton describes a model denoted by the acronym, DUET. Included is the effect of the lattice temperature, in addition to the electron and hole carrier temperatures. Comparisons with hydrodynamic models are discussed. The unifying theme of all of these models is their expression of mobilities as globally dependent quantities, rather than locally field dependent as in the drift diffusion case.

I should like to thank Professor George Zobrist for the invitation to organize this special issue, and for the encouragement he has provided. I hope that the readers of these papers will find them rewarding.

Joseph W. Jerome

\section{Biography}

JOSEPH W. JEROME received the Ph.D. degree in Mathematics from Purdue University in 1966. He was visiting Assistant Professor at the Mathematics Research Center, University of Wisconsin, during 1966-68, and was Assistant Professor at Case Western Reserve University during 1968-70. He joined Northwestern University in 1970, where he has been Professor of Mathematics and Applied Mathematics since 1976 . He has held sabbatical positions at Oxford University, England, 1974-75, University of Texas, 1978-79, and Bell Laboratories, Murray Hill, 1982-83. He was visiting scholar at the University of Chicago in 1985. His research interests include applied and numerical analysis, computational electronics, and cell membrane biology. His book on semiconductor modeling will be published by Springer in 1995 . 

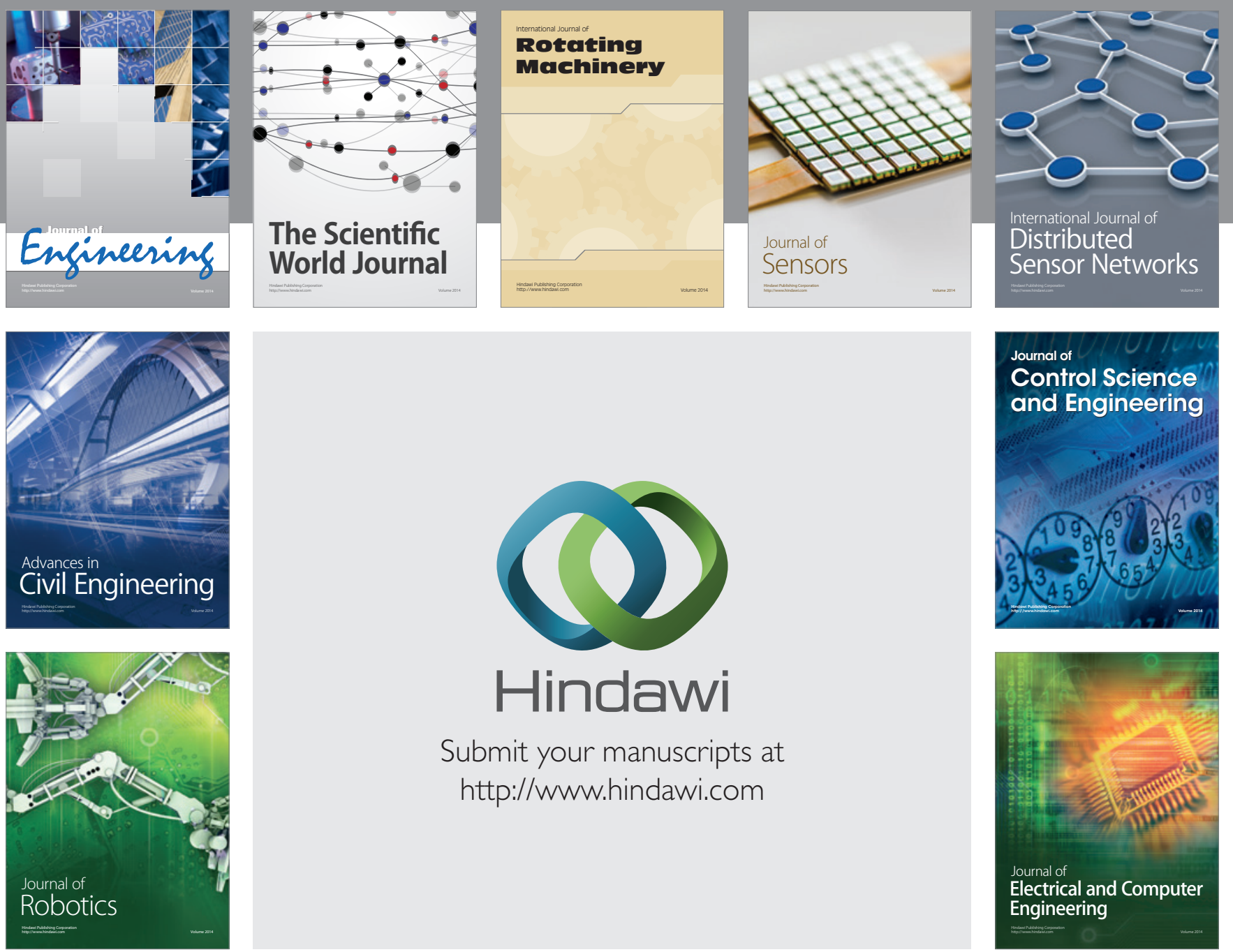

Submit your manuscripts at

http://www.hindawi.com
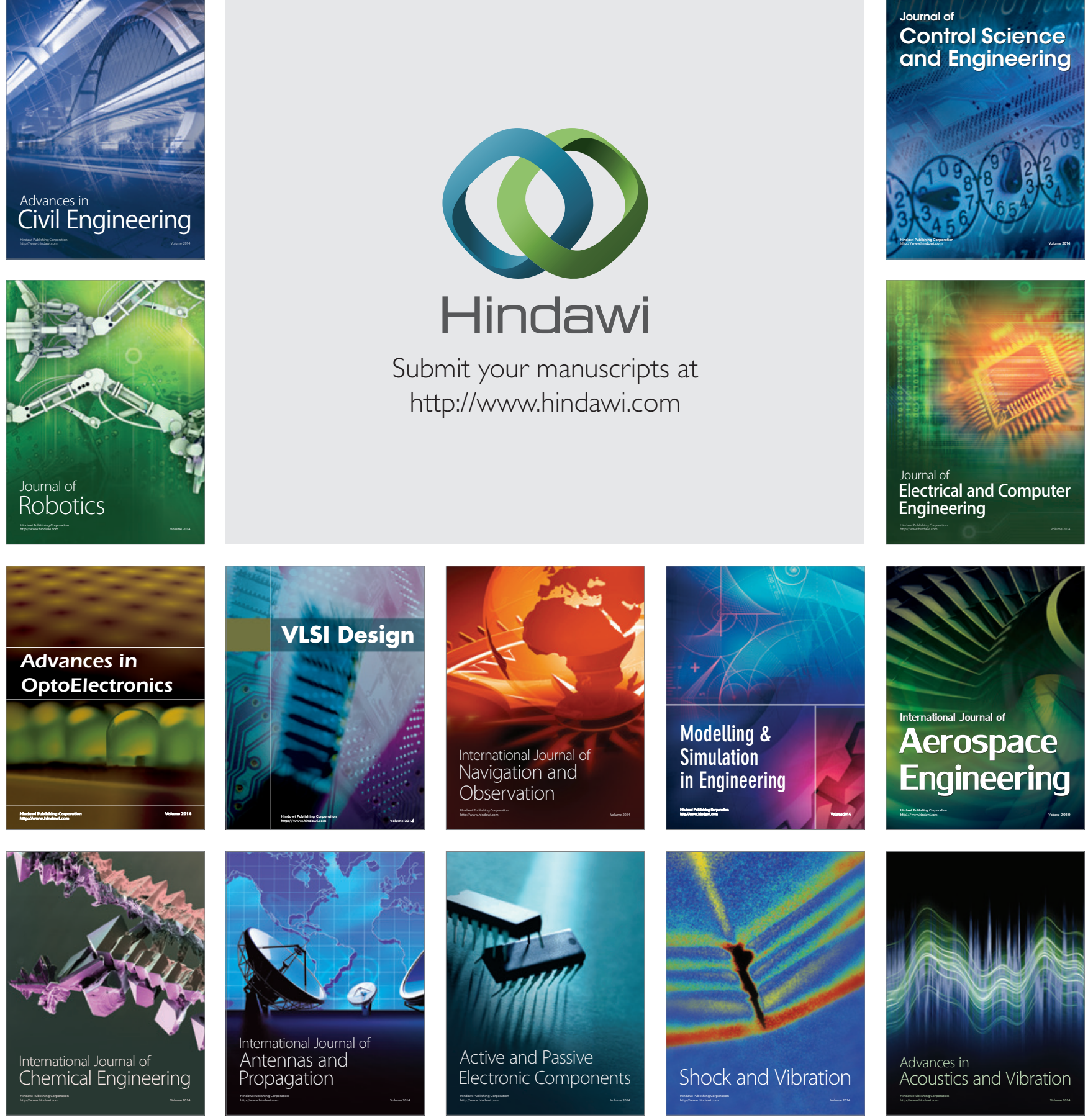